\title{
Tracheal torsion assessed by a computer-generated 3-dimensional image analysis predicts tracheal self-expandable metallic stent fracture
}

\author{
Chih-Teng Yu, MD, ${ }^{\text {a }}$ Chun-Liang Chou, MD, ${ }^{a}$ Fu-Tsai Chung, MD, ${ }^{\text {a Jei-Tsai Wu, MD, }}$ \\ Yuan-Chang Liu, MD, ${ }^{\mathrm{c}}$ Yun-Hen Liu, MD, ${ }^{\mathrm{d}}$ Ting-Yu Lin, MD, ${ }^{\mathrm{a}}$ Shu-Min Lin, MD, ${ }^{\mathrm{a}}$ \\ Horng-Chuang Lin, MD, ${ }^{a}$ Chun-Hua Wang, MD, ${ }^{\mathrm{a}}$ Han-Pin Kuo, MD, PhD, ${ }^{\mathrm{a}}$ Hao-Cheng Chen, MD, ${ }^{\mathrm{e}}$ and \\ Chien-Ying Liu, MD
}

\begin{abstract}
Objective: Self-expandable metallic stents are used to relieve airway stenosis in selected patients; however, fracture of these stents may occur. This analysis aims to investigate the extent of tracheal torsion, assessed by a computed-generated reformatted 3-dimensional tracheal reconstruction from 2-dimensional computed tomographic images in predicting fracture of tracheal self-expandable metallic stents.
\end{abstract}

\begin{abstract}
Methods: From 2001 to 2007, 32 patients (aged $62.8 \pm 14.1$ years) with benign tracheal diseases received chest computed tomographic evaluation and Ultraflex (Boston Scientific, Natick, Mass) self-expandable metallic stents. The bending angles of the central axis and peripheral wall of the trachea at choke point were measured from the computed-generated 3-dimensional tracheal images.
\end{abstract}

\begin{abstract}
Results: Seventeen fractured stents were found among the patients. The median time for stent fracture was 865 days after implantation. Receiver operating characteristic curve analysis revealed that a $19^{\circ}$ bending angle of the tracheal central axis (area under the curve, $0.929 ; 95 \%$ confidence interval, $0.847-1.012 ; P<.001$ ) and a $44^{\circ}$ maximal bending angle of the peripheral tracheal wall (area under the curve, $0.918 ; 95 \%$ confidence interval, $0.821-1.012 ; P<.001)$ had maximal power in predicting tracheal fracture of self-expandable metallic stents.

Conclusions: Three-dimensional tracheal reconstructions from 2-dimensional chest computed tomographic data are useful in assessing the severity of tracheal torsion. Tortuous trachea with a central axis bending angle of $19^{\circ}$ or more and peripheral tracheal wall maximal bending angle of $44^{\circ}$ or more were associated with a high probability of fracture of the self-expandable metallic stent. (J Thorac Cardiovasc Surg 2010;140:769-76)
\end{abstract}

Endoscopic airway stent implementation is effective in the management of benign and malignant airway diseases. In addition to silicone stents, ${ }^{1,2}$ self-expandable metallic stents (SEMSs) are widely used in the management of airway stenosis. They can be successfully implanted via a fiberoptic

\footnotetext{
From the Department of Thoracic Medicine, ${ }^{\text {a }}$ Medical Augmented Reality Research Center, ${ }^{\mathrm{b}}$ Department of Medical Imaging, ${ }^{\mathrm{c}}$ and Department of Thoracic Surgery, ${ }^{\mathrm{d}}$ Chang Gung Memorial Hospital, and School of Medicine, Chang Gung University,

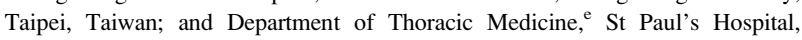
Tauyuan, Taiwan.

Financial support for this research was supplied by the National Science Council of the Republic of China, Taiwan, under contract Nos. NSC 97-2314-B-182A-091-MY2 and 95-2314-B-182A-018, and by the Chang-Gung Memorial Hospital, under contract Nos. CMRPG 371571 and 371651.

Disclosures: None.

C. T. Yu, C. L. Chou, and F. T. Chung contributed equally to the work on this project as first authors.

H. C. Chen and C. Y. Liu contributed equally to the work on this project as corresponding authors.

Received for publication Jan 7, 2010; revisions received April 6, 2010; accepted for publication April 25, 2010; available ahead of print June 25, 2010.

Address for reprints: Dr Chien-Ying Liu, Division of Interventional Bronchoscopy and Pulmonary Oncology, Department of Thoracic Medicine, Chang Gung Memorial Hospital and Chang Gung University, No. 199, Tun-Hwa North Rd, Taipei 105, Taiwan (E-mail: cyliu01@adm.cgmh.org.tw).

$0022-5223 / \$ 36.00$

Copyright (C) 2010 by The American Association for Thoracic Surgery

doi:10.1016/j.jtcvs.2010.04.025
}

bronchoscope while patients are under conscious sedation and local anesthesia. ${ }^{3-6}$ SEMSs adequately maintain the patency of a narrow airway caused by tracheobronchial diseases; however, complications, including migration, granulation tissue formation, impaired mucociliary clearance, recurrent stent obstruction, increased bacterial colonization and infection, and even stent fracture, may occur. $^{7,8}$ Therefore, the United States Food and Drug Administration warned that SEMS implantation should be considered only if patients are not eligible for surgical intervention, treatment with a rigid bronchoscopy, or silicone stent implantation. Although the use of SEMSs is discouraged in benign conditions, metallic stents have been reported to be safe and effective in the management of benign and malignant airway obstructions in patients who are not suitable for surgical intervention because of medical comorbidities or poor pulmonary function. ${ }^{9-13}$

Among the potential complications of SEMSs, fracture is of major concern because it can produce airway obstruction and wall perforation. ${ }^{14}$ Repetitive coughing, esophageal compression during swallowing, ${ }^{7}$ metal fatigue, ${ }^{3}$ granulation, and shearing forces ${ }^{15}$ are the proposed causes of such fractures. However, our previous study revealed that a tortuous airway is the independent factor contributing to SEMS 


$$
\begin{aligned}
& \text { Abbreviations and Acronyms } \\
& \begin{aligned}
95 \% \mathrm{CI} & =95 \% \text { confidence interval } \\
\mathrm{CT} & =\text { computed tomography } \\
\text { 2D } & =2 \text {-dimensional } \\
\text { 3D } & =3 \text {-dimensional } \\
\text { ROC } & =\text { receiver operating characteristic curve } \\
\text { SEMS } & =\text { self-expandable metallic stent }
\end{aligned}
\end{aligned}
$$

fracture. ${ }^{16}$ Therefore, the development of a method to estimate the extent of tracheal torsion is critical to identify patients who are at high risk for fracture.

Hardware and software evolution has facilitated the 2-dimensional (2D) reformatting of chest computed tomography (CT) data sets and the 3-dimensional (3D) reconstruction of thoracic structures. ${ }^{17}$ Additional rendering allows precise evaluation of the morphology, length, degree of stenosis, and extent of torsion in the central airway. ${ }^{17}$ In the present study, we further applied 3D CT imaging technique, developed in the Medical Augmented Reality Research Center in our hospital, to evaluate the extent of tracheal torsion and its relationship with tracheal SEMS fracture in patients with stenotic trachea.

\section{PATIENTS AND METHODS \\ Patients}

From January 2001 to December 2007, 32 patients with a mean age of $62.8 \pm 14.1$ years who underwent endoscopic tracheal stent placement for the treatment of benign tracheal diseases at Chang-Gung Memorial Hospital, a university-affiliated hospital in Taiwan, and had chest CT studies before the procedure were enrolled for analysis. Silicone or other metallic stents were ordinarily used by thoracic surgeons at our institution; however, most of them require intervention with a rigid bronchoscope. Thoracic surgeons were routinely consulted regarding the feasibility of surgical intervention for all the benign diseases before SEMS implantation. The enrolled patients were not candidates for surgical intervention because of their poor pulmonary function or severe comorbid conditions. Informed consent was obtained from all patients or their surrogates before bronchoscopic SEMS implantation. Methodology and patient confidentiality were approved by our institutional review board. The design of the retrospective study was approved by the institutional review board (CGMH IRB No. 95-1409B).

\section{Stent Implantation and Follow-up Assessment}

The location, length, and severity of stenosis (grade I, $<50 \%$; grade II, $51 \%-70 \%$; grade III, $71 \%-99 \%$ ) were determined on the basis of the CT images and bronchoscopic studies before the implantation. SEMSs were implanted by flexible fiberoptic bronchoscopes with local anesthesia and conscious sedation. The choice of stent size, length, and type (with or without cover) was determined by the findings of previous endoscopic examination and chest CT studies. SEMSs were implanted at the choke point, as determined by using a flow-volume curve, bronchoscopic endobronchial ultrasonography, or 3D CT before stent implantation. The Ultraflex stent (Boston Scientific, Natick, Mass), a tightly woven SEMS composed entirely of a single strand of nickel-titanium alloy, was the stent used in this study. A second bronchoscopic study was performed 48 hours after the implantation. Further bronchoscopic follow-up studies were performed 1 week after im- plantation and every 3 to 6 months thereafter. If dyspnea, severe coughing, increased mucus production, or other fracture symptoms occurred, additional bronchoscopic analysis was performed.

\section{Reconstruction of 3D Tracheal Images and Measurement of the Central Axis and Lateral Wall Bending Angles at the Tracheal Choke Point}

The raw data sets of the CT sections were used for the analysis. After the 2D image postprocessing and 3D reconstruction using the software developed in the Medical Augmented Reality Research Center, the tracheal $3 \mathrm{D}$ structure was rendered. The central axis bending angle at the previously determined tracheal choke point and the maximal bending angle of the peripheral wall at that location were calculated by using the software.

\section{Statistical Analysis}

Descriptive statistics were used to examine the demographic characteristics of the study population. Data were presented as mean \pm standard deviation. For univariate analyses, Mann-Whitney $U$ test was used to compare data between groups. Differences in categorical data were analyzed using the $\chi^{2}$ or Fisher's exact tests. The odds ratio and $95 \%$ confidence intervals $(95 \% \mathrm{CI})$ or likelihood ratio were used to assess the independent contribution of significant variables. Correlations between variables were analyzed by the Spearman ranking test. The receiver operating characteristic curve (ROC) was used to analyze the discrimination threshold of the varied cutoff values of the tracheal central axis and peripheral wall bending angles. Time to SEMS fracture was defined as the time between the date of implantation and fracture detection. Kaplan-Meier analysis was used to determine the SEMS fracture rate curve. Incidence curves of SEMS fracture between patients with different tracheal torsion severities were compared by using the log-rank test. All analyses were performed using SPSS software version 10.0 (SPSS, Inc, Chicago, Ill) and GraphPad Prism 3.0 (GraphPad Software, San Diego, Calif).

TABLE 1. Characteristics of patients with tracheal SEMS placement

\begin{tabular}{lc}
\hline \multicolumn{1}{c}{ Characteristic } & Total \\
\hline Patient (no.) & 32 \\
Fracture/nonfracture & $15 / 17$ \\
Age (y) & $62.8 \pm 14$. \\
Gender, male/female (no.) & $26 / 6$ \\
Causes of stenosis and malacia & \\
Corrosive injury & 3 \\
Postintubation stricture & 13 \\
Posttuberculosis stricture & 12 \\
Granulomatous formation & 2 \\
Goiter compression & 2
\end{tabular}

Pulmonary function test before procedure

FVC (L)

$1.81 \pm 0.76$

FVC $(\%)$

$\mathrm{FEV}_{1}(\mathrm{~L})$

$\mathrm{FEV}_{1}(\%)$

$54.8 \pm 18.3$

$1.14 \pm 0.63$

$47.28 \pm 15.6$

Choke point for SEMS placement site (no.)

Upper

14

Middle

8

Lower

10

Stenotic length $(\mathrm{cm})$

$1.62 \pm 0.56$

Stenotic severity (grade)

$2.31 \pm 0.51$

Stent size (OD, mm)

$18.06 \pm 2.53$

Stent length $(\mathrm{cm})$

$6.28 \pm 0.76$

$S E M S$, Self-expandable metallic stent; $F V C$, forced vital capacity; $F E V_{l}$, forced expiratory volume in 1 second; $O D$, outer diameter. 



FIGURE 1. Tracheal torsion and choke point were detected by chest radiography (A) and fiberoptic bronchoscopy (B). To estimate the tracheal maximal bending angle at choke point, 2D images of chest CT (C) were reformatted for the computer-generated 3D CT analysis. A volume-rendered 3D image of the trachea (D) was constructed. Data represent 1 study subject. $C T$, Computed tomography; $2 D$ and 3D, 2- and 3-dimensional.

\section{RESULTS}

\section{Patient Baseline Characteristics}

From January 2001 to December 2007, 32 SEMS implantation procedures involving 32 patients were analyzed. Patient characteristics are presented in Table 1. Tracheal stenosis was due to corrosive injury in 3 patients, postintubation stricture in 13 , posttuberculosis stricture in 12 , granulomatous formation and lumen narrowing in 2, and goiter compression in 2 patients. Before SEMS placement, the mean forced vital capacity of the patients was $1.81 \pm 0.76$ $\mathrm{L}(54.8 \% \pm 18.3 \%$ of the normal predicted value) and the mean forced expiratory volume in 1 second was $1.14 \pm$ $0.63 \mathrm{~L}(47.28 \% \pm 15.6 \%$ of the normal predicted value). SEMSs were placed in the upper third of the trachea in 14 patients, in the middle third in 8 , and in the lower third in 10. The mean length of stenosis was $1.62 \pm 0.56 \mathrm{~cm}$. The mean grade of tracheal stenosis was $2.31 \pm 0.51$ (range $2-3)$. The size of SEMSs ranged from 16 to $20 \mathrm{~mm}$ $(18.06 \pm 2.53 \mathrm{~mm})$ in outer diameter $\times 4$ to $8 \mathrm{~cm}(6.28 \pm$ $0.76 \mathrm{~cm}$ ) in length. SEMS fracture occurred in 17 patients during the follow-up period (Table 1).

\section{Volume-Rendered 3D Image of the Trachea Was Reconstructed to Measure the Extent of Tracheal Torsion at Choke Point}

From the finding that tracheal torsion at the choke point, as detected by chest radiography and fiberoptic bronchoscopy (Figure 1, $A$ and $B$ ), is an independent factor contributing 


\section{Principle of Bending Angle Measurement}

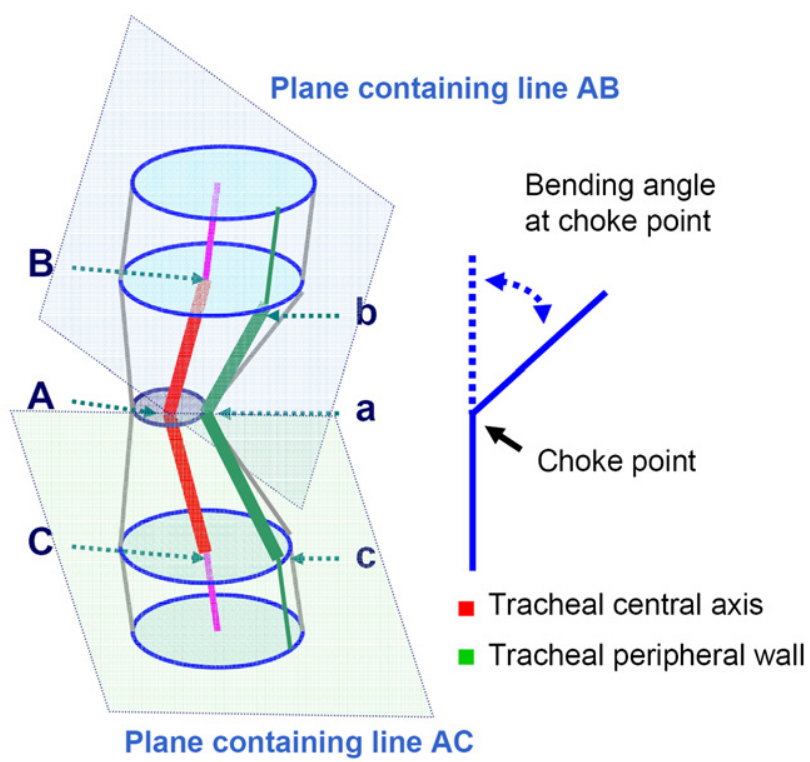

FIGURE 2. Model for measuring the maximal bending angles of tracheal central axis and peripheral wall at the choke point. A 3D tracheal structure was reconstructed from the $2 \mathrm{D}$ chest tomography, and the choke point level (point $A$ ) of tracheal stenosis was first determined according to the bronchoscopic and the CT findings. The central axis above the choke point (line $A B$ ) and the axis below the choke point (line $A C$ ) were determined, and an angle (angle $B A C$ ) was formed. The bending angle of the central axis at choke point can be defined as 180-angle BAC in degrees. Using similar principles, the borders of the tracheal rings in all 2D sections were reconstructed to form a $3 \mathrm{D}$ structure of the tracheal wall. The planes containing the central axis above or below the choke point (line $A B$ or $A C$ ) will intersect the tracheal wall and form numerous cutting lines on the tracheal wall above and below the choke point level (eg, line $a b$ or line $a c$ ). Then, the peripheral wall angle at the choke point level could be calculated (eg, angle bac). The bending angle of peripheral wall can also be defined as 180 -angle bac in degrees. The axial bending angle and the maximal peripheral wall bending angle at the choke point level were calculated by the software created in our center.

to SEMS fracture, ${ }^{16}$ the original $2 \mathrm{D}$ chest $\mathrm{CT}$ images (Figure 1,C) were further reconstructed to create a 3D structural image of the trachea (Figure 1,D). Using the geometric calculation program in the 3D imaging software, we calculated the central axis bending angle and the maximal peripheral wall bending angle at the tracheal choke point (Figure 2).

\section{Tracheal Bending Angle Differences at the Choke Point in Patients With and Without SEMS Fracture}

Our analysis indicated a correlation between the maximal bending angles of the central axis and peripheral wall of the trachea at the choke point $(\mathrm{n}=32 ; r=0.8491 ; 95 \% \mathrm{CI}$, $0.7054-0.9257 ; P<.0001$; Figure $3, A$ ). The maximal bending angles at the tracheal choke point in patients with SEMS fracture was $34.8^{\circ} \pm 13.5^{\circ}$ for the central axis and $61.5^{\circ} \pm 19.6^{\circ}$ for the peripheral wall. The extent of the tracheal torsion was significantly greater than in patients without SEMS fracture
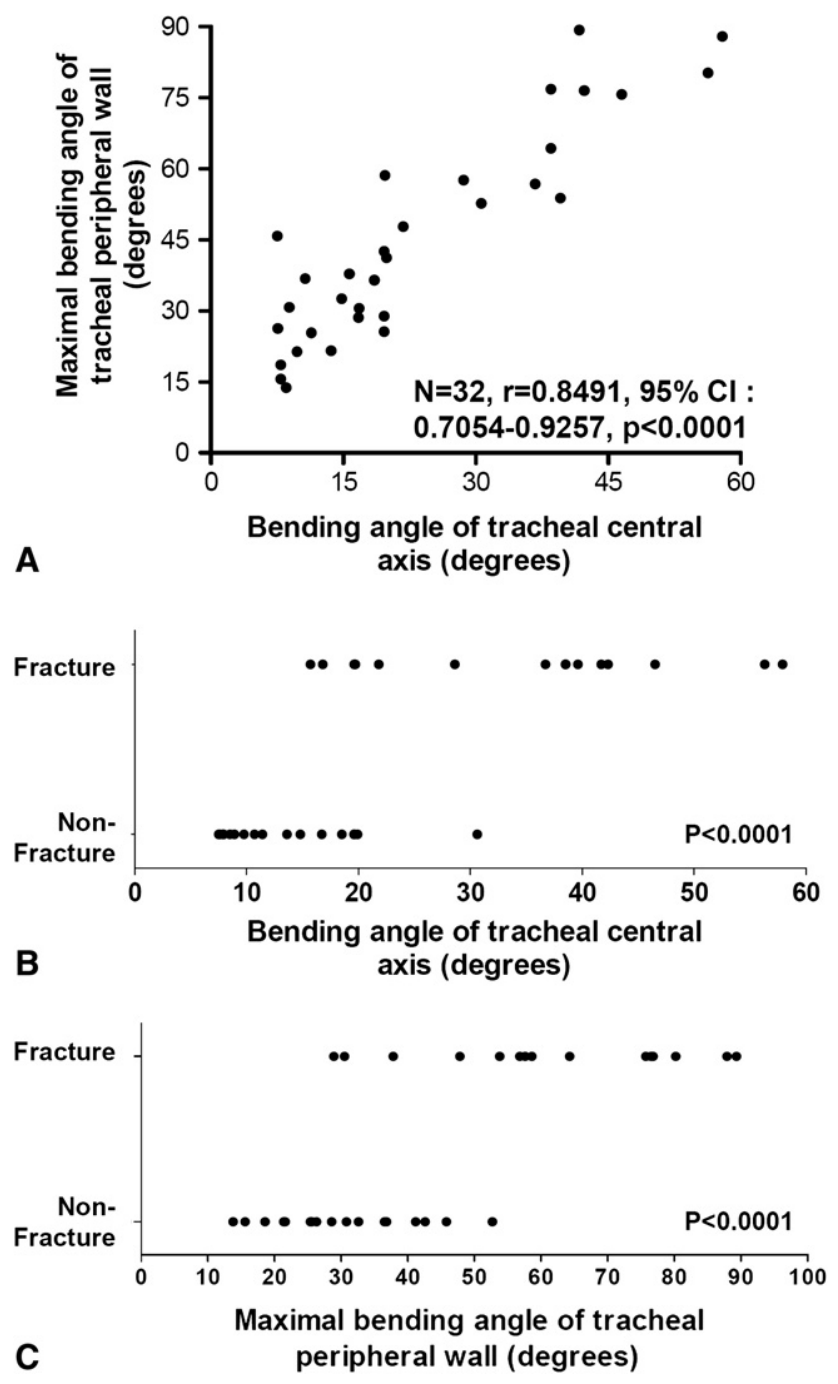

FIGURE 3. The correlation between the maximal bending angles of the tracheal central axis and peripheral wall (A). The occurrence of SEMS fracture and the bending angle of the tracheal central axis (B), and the maximal bending angle of the tracheal peripheral wall (C). Each point corresponds to a study subject. Spearman ranking test was used. $C I$, Confidence interval.

in whom the maximal bending angle of the tracheal central axis was $13.7^{\circ} \pm 6.4^{\circ}(P<.0001$; Figure $3, B)$ and that of the tracheal peripheral wall was $30.3^{\circ} \pm 11.0^{\circ}(P<.0001$; Figure 3,C).

\section{Cutoff Values of the Maximal Bending Angle at the Tracheal Choke Point Were Determined by ROC Analysis}

ROC analysis was performed to further determine the maximal bending angle of the trachea at the choke point that would allow predicting SEMS fracture with maximal accuracy. This analysis revealed that a cutoff value of $19^{\circ}$ tracheal central axis bending angle resulted in a sensitivity of 0.800 and a specificity of 0.882 , corresponding to maximal discriminative power (area under the curve, 0.929; 
Coordinates of the Curve

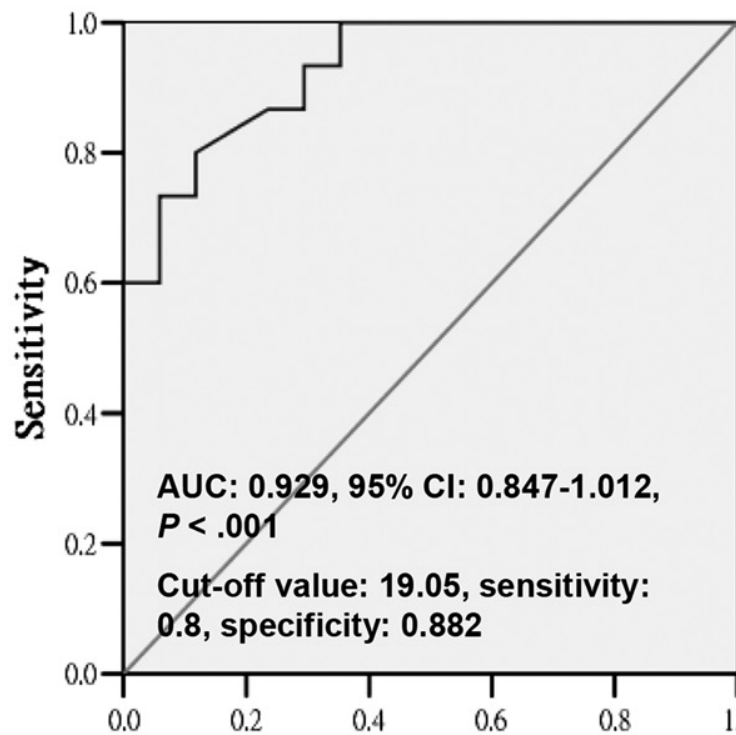

Angle (degrees) Sensitivity Specificity

Sum

\begin{tabular}{rrrr}
\hline 15.25 & 1.000 & 0.647 & 1.647 \\
16.20 & 0.933 & 0.647 & 1.580 \\
16.75 & 0.933 & 0.706 & 1.639 \\
17.65 & 0.867 & 0.765 & 1.632 \\
$\mathbf{1 9 . 0 5}$ & $\mathbf{0 . 8 0 0}$ & $\mathbf{0 . 8 8 2}$ & $\mathbf{1 . 6 8 2}$ \\
20.85 & 0.733 & 0.941 & 1.647 \\
25.20 & 0.667 & 0.941 & 1.618 \\
33.65 & 0.600 & 1.000 & 1.600 \\
\hline
\end{tabular}

A

1 - Specificity

Coordinates of the Curve

\begin{tabular}{|c|c|c|c|c|c|}
\hline \multirow[b]{2}{*}{$0.8-$} & & Angle (degrees) & Sensitivity & Specificity & Sum \\
\hline & & 28.7 & 1.000 & 0.529 & 1.529 \\
\hline & & 30.6 & 0.867 & 0.529 & 1.396 \\
\hline & & 34.5 & 0.867 & 0.647 & 1.514 \\
\hline $0.4-$ & & 37.3 & 0.867 & 0.765 & 1.632 \\
\hline & AUC: $0.918,95 \%$ Cl: $0.821-1.012$, & 39.5 & 0.800 & 0.765 & 1.565 \\
\hline $0.2-$ & Cut-off value: 44.2 , sensitivity: 0.8 , & 44.2 & 0.800 & 0.882 & 1.682 \\
\hline & specificity: 0.882 & 50.3 & 0.733 & 0.941 & 1.674 \\
\hline 0.07 & $T_{06}$ & 55.3 & 667 & 1.000 & 1.667 \\
\hline
\end{tabular}

B

1 - Specificity

FIGURE 4. Receiver operating characteristic curve analysis of the maximal bending angle at the tracheal choke point and the occurrence of SEMS fracture. A, Bending angle of the tracheal central axis. B, Maximal bending angle of the tracheal peripheral wall. The diagonal line indicates no discrimination; the staircase line indicates the empirical curve. Figures in bold type of the coordinates of the curve, with the corresponding sensitivity and specificity values, denote the cutoff values with best discriminative power. $C I$, Confidence interval; $A U C$, area under the curve.

standard error, $0.042 ; 95 \%$ CI, 0.847-1.012; $P<.001$; Figure $4, A$ ). A cutoff value of $44^{\circ}$ maximal bending angle of the peripheral wall at the tracheal choke point was found to have a sensitivity of 0.800 and a specificity of 0.882 , corresponding to maximal discriminative power (area under the curve, 0.918; standard error, 0.049; 95\% CI, 0.821-1.012; $P<.001 ;$ Figure $4, B)$.
Extent of Tracheal Torsion Predicted SEMS Fracture

Further application of the cutoff values revealed that a tracheal central axis angle of $19^{\circ}$ or more was associated with an odds ratio of $21.13(95 \% \mathrm{CI}, 3.276-136.2 ; P=.0005)$ and a peripheral wall maximal bending angle of $44^{\circ}$ or more was associated with an odds ratio of $30.0(95 \% \mathrm{CI}$, 4.294-209.6; $P=.0002$; Table 2) for risk of SEMS fracture. 
TABLE 2. Univariate analysis of the probability of tracheal SEMS fracture in patients subgrouped according to the cutoff value of bending angle determined by ROC analysis

\begin{tabular}{lccccc}
\hline $\begin{array}{c}\text { Tracheal torsion } \\
\text { at the choke point }\end{array}$ & Fracture & Nonfracture & $\begin{array}{c}\text { Odds } \\
\text { ratio }\end{array}$ & $\mathbf{9 5 \%}$ CI & $\begin{array}{c}P \\
\text { value }\end{array}$ \\
\hline Bending angle of tracheal central axis (degrees) & & \\
$\geq 19$ & 13 & 4 & 21.13 & $3.276-136.2$ & .0005 \\
$<19$ & 2 & 13 & & & \\
Maximal bending angle of tracheal peripheral wall (degrees) & \\
$\geq 44$ & 12 & 2 & 30.00 & $4.294-209.6$ & .0002 \\
$<44$ & 3 & 15 & & & \\
\hline
\end{tabular}

$\overline{S E M S \text {, Self-expandable metallic stent; } R O C \text {, receiver operating characteristic curve; }}$ $C I$, confidence interval.

Moreover, logistic regression analysis of cumulative SEMS fracture in patients with varying extents of tracheal torsion revealed that the median time to SEMS fracture was 687 days in patients with a central axis bending angle of $19^{\circ}$ or more (hazard ratio, 3.113; 95\% CI, 1.066-7.670; $P=.0368)$, compared with patients with an angle of less than $19^{\circ}$ (Figure 5, A). The median time to SEMS fracture was 681 days in patients with a $44^{\circ}$ or greater maximal bending angle of the peripheral tracheal wall (hazard ratio, 4.656; $95 \% \mathrm{CI}, 1.422-10.820 ; P=.0084)$, compared with patients with an angle of less than $44^{\circ}$ (Figure $5, B$ ).

\section{DISCUSSION}

In this study, we applied the 3D reconstruction postprocessing technique to raw 2D thoracic CT-section data. The tracheal 3D images were reconstructed, and the extent of tracheal torsion was accurately determined by measuring the bending angles of the tracheal central axis and peripheral wall. We demonstrated that the maximal bending angles between the central axis and peripheral wall of the trachea at the choke point were relevant. Cutoff values of $19^{\circ}$ for the bending angle of the central axis and $44^{\circ}$ for the maximal bending angle of the peripheral wall had maximal power in discriminating the risk of SEMS fracture. Bending angles larger than those values were associated with SEMS fracture.

Hardware and software evolution has facilitated the 2D reformatting of chest CT data sets and the 3D reconstruction of thoracic structures. ${ }^{17}$ 3D imaging modality has provided a novel method for the evaluation of airway diseases and facilitated pulmonary clinical investigation and practice. ${ }^{18,19}$ Recent advances in multiple detector rows and rapid spiral thin-section techniques have facilitated the diagnostic evaluation of pathologic processes affecting the airways. ${ }^{18,20,21}$ Moreover, better longitudinal resolution can provide accurate investigation of distal bronchi in section planes orthogonal to their axes, and quantitative 3D CT virtual bronchography can be further reconstructed. ${ }^{18,19,22-24}$ Additional rendering techniques will allow precise evaluations of the morphology, length, degree of stenosis, and extent of torsion in the central

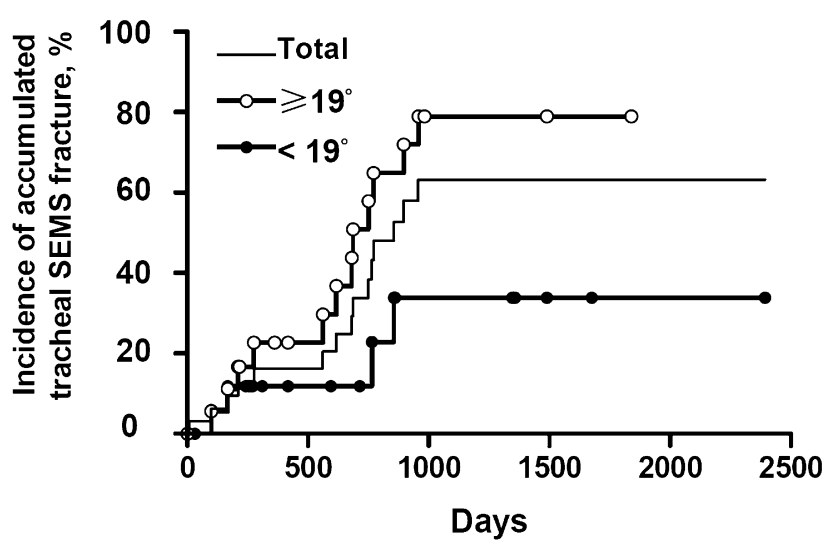

A

Log rank test $p=0.0368$,

Hazard ratio $3.113,95 \% \mathrm{Cl}: 1.066-7.670$

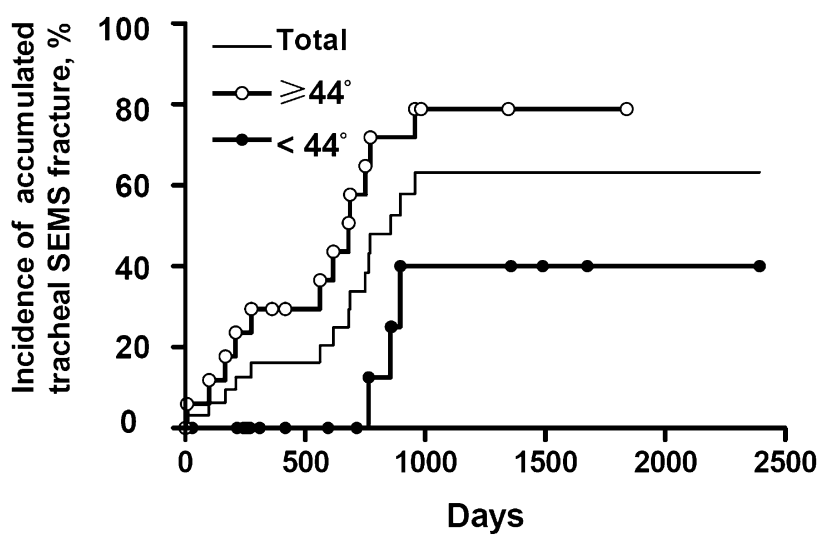

B Hazard ratio $4.656,95 \% \mathrm{Cl}: 1.422-10.820$

FIGURE 5. Logistic regression analysis of cumulative SEMS fracture incidence in patients with varying extents of tracheal torsion. A, Bending angle of the tracheal central axis at the choke point. Median time to tracheal SEMS fracture was 687 days in angles of $19^{\circ}$ or more. B, Maximal bending angle of the tracheal peripheral wall at the choke point. Median time to SEMS fracture was 681 days in angles of $44^{\circ}$ or more. SEMS, Selfexpandable metallic stent.

airway. ${ }^{17}$ Moreover, with improvements in the display of the relationships between mucosal structures and bronchovascular interfaces, the locoregional extent of mucosal abnormalities, such as central tumors and focal or diffuse airway diseases, can be accurately depicted, ${ }^{18,20,21}$ increasing the synergy between CT and bronchoscopy. In addition, dynamic and functional evaluation can be achieved with increased speeds of spiral scanning.

The 3D image processing software and techniques developed in our center can construct 3D images from the raw data set of traditional 2D CT scans of the thorax and render the tracheal structures. However, in this study, most of the patients underwent the CT examination before our hospital had multiple detector row rapid spiral thin-section CT scanner consoles. The extent of tracheal torsion and the severity 
of angulation could be accurately calculated, and the choke point of the trachea could be well defined in combination with bronchoscopic findings; however, the detailed tracheal wall structures could not be rendered because of the limited resolution of the original $\mathrm{CT}$ images.

A previous study has shown that SEMSs improve quality of life of patients with malignant lung disease complicated with central airway narrowing. ${ }^{16}$ Because of the limited life expectancy, SEMS fracture is seldom seen in these patients. ${ }^{17}$ In patients with benign narrowed airway, the incidence of SEMS fracture increases after 2 years in patients with tortuous airways. ${ }^{17}$ In addition to increased shearing forces on the stents, granulation tissue formation, repetitive coughing, metal failure, and compression from the esophagus during swallowing have been proposed as the causes of stent fracture. ${ }^{3,7,15}$ Our previous study revealed that stent length and size, as well as the site of implantation, were not associated with SEMS fracture. ${ }^{17}$ The structural characteristics of a tortuous airway and postoperative airway conditions (poor alignment, incomplete stent lumen expansion, granulation tissue formation, and narrow stent opening) are related to stent fracture. Our previous study had further revealed that patients with tortuous airways also had a higher incidence of granulation tissue formation, incomplete stent lumen expansion, poor airway alignment, and narrow stent opening when compared with those without airway tortuosity. ${ }^{17}$ Furthermore, an underlying tortuous airway had been proven to be the only independent predictor of stent fracture. ${ }^{17}$

In the present study, owing to the development of $2 \mathrm{D}$ image postprocessing techniques and $3 \mathrm{D}$ reconstruction and formatting software in our center, the bending angles of the central axis and peripheral wall of the trachea at the choke point were calculated. The bending angle of $19^{\circ}$ for tracheal axis had a sensitivity of 0.800 and a specificity of 0.882 , and the maximal bending angle of $44^{\circ}$ for the peripheral tracheal wall had a sensitivity of 0.800 and a specificity of 0.822 , in predicting the occurrence of SEMS fracture. For the tracheal tubular structure, the 2 angles are highly correlated. These findings suggest that SEMS placement in benign tracheal diseases should be executed with caution to avoid the risk of fracture. For patients with benign airway diseases complicated with stable or dynamic narrowing, surgery and non-SEMS placement should be the first choice of treatment. If patients are not suitable for surgical intervention because of poor lung function, comorbidities, or refusal of surgery, SEMS implantation using a flexible fiberoptic bronchoscope may be considered. Our previous data showed that there was no significant change in the incidence of SEMS fracture with time in patients with nontortuous airways. Therefore, evaluation of the extent and severity of airway torsion is critically important. Multiplerow, multislice CT can cover the whole thoracic volume in a single breath hold and should be helpful in evaluation before the procedure. Its speed and high resolution can facilitate the precise assessment of anatomic structures and in dynamic airway studies. SEMS implantation may still be useful for patients without or with less severe tracheal torsion. Regular radiologic or bronchoscopic examination and close monitoring of symptoms such as the new development of dyspnea or coughing after SEMS implantation is mandatory. ${ }^{17}$

In conclusion, SEMS implantation should be performed cautiously in patients with benign central airway lesions and tortuous airways. Using 3D reconstruction technique and image analysis, the morphologic characteristics of airway structure, including the extent of torsion and bending angles at choke point, can be precisely evaluated. SEMS should be restricted to a highly selected population. For patients with a tracheal axis bending angle of $19^{\circ}$ or more and a peripheral wall maximal bending angle of $44^{\circ}$ or more, SEMS placement should be avoided.

\section{References}

1. Martinez-Ballarin JI, Diaz-Jimenez JP, Castro MJ, Moya JA. Silicone stents in the management of benign tracheobronchial stenoses. Tolerance and early results in 63 patients. Chest. 1996;109:626-9.

2. Wood DE, Liu YH, Vallieres E, Karmy-Jones R, Mulligan MS. Airway stenting for malignant and benign tracheobronchial stenosis. Ann Thorac Surg. 2003;76: 167-72.

3. Aggarwal A, Dasgupta A, Mehta AC. Metalloptysis expulsion of wire stent fragments. Chest. 1999;115:1484-5.

4. Noppen M, Meysman M, Claes I, D'Haese J, Vincken W. Screw-thread vs Dumon endoprosthesis in the management of tracheal stenosis. Chest. 1999; 115:532-5.

5. Madden BP, Datta S, Charokopos N. Experience with Ultraflex expandable metallic stents in the management of endobronchial pathology. Ann Thorac Surg. 2002;73:938-44.

6. Saad CP, Murthy S, Krizmanich G, Mehta AC. Self-expandable metallic airway stents and flexible bronchoscopy: long-term outcomes analysis. Chest. 2003;124: 1993-9.

7. Zakaluzny SA, Lane JD, Mair EA. Complications of tracheobronchial airway stents. Otolaryngol Head Neck Surg. 2003;128:478-88.

8. Gaissert HA, Grillo HC, Wright CD, Donahue DM, Wain JC, Mathisen DJ. Complication of benign tracheobronchial strictures by self-expanding metal stents. J Thorac Cardiovasc Surg. 2003;126:744-7.

9. Madden BP, Loke TK, Sheth AC. Do expandable metallic airway stents have a role in the management of patients with benign tracheobronchial disease? Ann Thorac Surg. 2006;82:274-8.

10. Husain SA, Finch D, Ahmed M, Morgan A, Hetzel MR. Long-term follow-up of Ultraflex metallic stents in benign and malignant central airway obstruction. Ann Thorac Surg. 2007;83:1251-6.

11. Nashef SA, Dromer C, Velly JF, Labrousse L, Couraud L. Expanding wire stents in benign tracheobronchial disease: indications and complications. Ann Thorac Surg. 1992;54:937-40.

12. Ducic Y, Khalafi RS. Use of endoscopically placed expandable nitinol tracheal stents in the treatment of tracheal stenosis. Laryngoscope. 1999;109: 1130-3.

13. Vergnon JM, Costes F, Bayon MC, Emonot A. Efficacy of tracheal and bronchial stent placement on respiratory functional tests. Chest. 1995;107:741-6.

14. Rousseau H, Dahan M, Lauque D, Carre P, Didier A, Bilbao I, et al. Selfexpandable prostheses in the tracheobronchial tree. Radiology. 1993;188: 199-203.

15. Burningham AR, Wax MK, Andersen PE, Everts EC, Cohen JI. Metallic tracheal stents: complications associated with long-term use in the upper airway. Ann Otol Rhinol Laryngol. 2002;111:285-90.

16. Chung FT, Lin SM, Chen HC, Chou CL, Yu CT, Liu CY, et al. Factors leading to tracheobronchial self-expandable metallic stent fracture. $J$ Thorac Cardiovasc Surg. 2008;136:1328-35. 
17. Salvolini L, Bichi Secchi E, Costarelli L, De Nicola M. Clinical applications of 2D and 3D imaging of the airways-a review. Eur J Radiol. 2000;34: 9-25.

18. Fetita CI, Prêteux F, Beigelman-Aubry C, Grenier P. Pulmonary airways: 3-D reconstruction from multislice $\mathrm{CT}$ and clinical investigation. IEEE Trans Med Imaging. 2004;23:1353-64.

19. Senéterre E, Paganin F, Bruel JM, Michel FB, Bousquet J. Measurement of internal size of bronchi using high-resolution computerized tomography (HRCT). Eur Respir J. 1994;7:596-600.

20. Sonka M, Park W, Hoffman EA. Rule-based detection of intrathoracic airway trees. IEEE Trans Med Imaging. 1996;15:314-26.
21. Tozaki T, Kawata Y, Niki N, Ohmatsu H, Eguchi K, Moriyama N. Three-dimensional analysis of lung areas using thin-slice CT images. Proc IEEE. 1996;3:548-52.

22. Brillet PY, Fetita CI, Capderou A, Mitrea M, Dreuil S, Simon JM, et al. Variability of bronchial measurements obtained by sequential CT using two computer-based methods. Eur Radiol. 2009;19:1139-47.

23. Brillet PY, Fetita CI, Saragaglia A, Brun AL, Beigelman-Aubry C, Prêteux F, et al. Investigation of airways using MDCT for visual and quantitative assessment in COPD patients. Int J Chron Obstruct Pulmon Dis. 2008;3:97-107.

24. Horton KM, Horton MR, Fishman EK. Advanced visualization of airways with 64-MDCT: 3D mapping and virtual bronchoscopy. AJR Am J Roentgenol. 2007;189:1387-96. 